\title{
Positive Fecal Occult Blood Test is a Predictive Factor for Gastrointestinal Bleeding after Capsule Endoscopy in Patients with Unexplained Iron Deficiency Anemia: A Korean Multicenter CAPENTRY Study
}

\author{
Ji Young Chang', Chang Mo Moon', Ki-Nam Shim', Dae Young Cheung ${ }^{2}$, Hyun Seok Lee ${ }^{3}$, Yun Jeong Lim", Seong Ran Jeon ${ }^{5}$, Soo \\ Jung Park ${ }^{6}$, Kyeong Ok Kim${ }^{7}$, Hyun Joo Song ${ }^{8}$, Hyun Joo Jang ${ }^{9}$ and Ji Hyun Kim ${ }^{10}$ \\ ${ }^{1}$ Department of Internal Medicine, Ewha Womans University College of Medicine, Seoul, ${ }^{2}$ Department of Internal Medicine, The Catholic \\ University of Korea College of Medicine, Seoul, ${ }^{3}$ Department of Internal Medicine, School of Medicine, Kyungpook National University, \\ Kyungpook National University Hospital, Daegu, ${ }^{4}$ Department of Internal Medicine, Dongguk University Ilsan Hospital, Goyang, \\ ${ }^{5}$ Department of Internal Medicine, Soonchunhyang University College of Medicine, Seoul, ${ }^{6}$ Department of Internal Medicine and \\ Institute of Gastroenterology, Yonsei University College of Medicine, Seoul, ${ }^{7}$ Department of Internal Medicine, Yeungnam University \\ College of Medicine, Daegu, ${ }^{8}$ Department of Internal Medicine, Jeju National University School of Medicine, Jeju, ${ }^{9}$ Department of \\ Internal Medicine, Hallym University College of Medicine, Hwasung, ${ }^{10}$ Department of Internal Medicine, Busan Paik Hospital, Inje \\ University College of Medicine, Busan, Korea
}

Background/Aims: Capsule endoscopy (CE) is recommended as the primary method for the evaluation of unexplained anemia. This study aimed to assess the diagnostic yield of CE in patients with unexplained iron deficiency anemia (IDA) without overt bleeding, and to evaluate their long-term outcomes and related clinical factors.

Methods: Data of patients who underwent CE for the evaluation of IDA were reviewed from a CE registry in Korea. Additional clinical data were collected by the involved investigators of each hospital through a review of medical records.

Results: Among a total of 144 patients, the diagnostic yield of CE was 34\%. Gastrointestinal (GI) bleeding was found in 6.3\% ( $n=9$ ) of the patients (occult bleeding in four patients and overt bleeding in five patients) during a mean follow-up of 17.8 months. Patients with a positive fecal occult blood test (FOBT) result at the initial diagnosis had a higher rate of GI bleeding after CE ( $p=0.004)$. In addition, a positive FOBT result was the only independent predictive factor for GI bleeding (hazard ratio, 5.30; 95\% confidence interval, $1.41-19.85 ; p=0.013)$.

Conclusions: Positive FOBT is a predictive factor for GI bleeding during follow-up after CE in patients with unexplained IDA without overt bleeding. Thus, patients with positive FOBT need to be more closely followed up. Clin Endosc 2020;53:719-726

Key Words: Capsule endoscopy; Fecal occult blood test; Gastrointestinal bleeding; Iron deficiency anemia; Predictive factors

Received: July 30, 2019 Revised: May 14, 2020

Accepted: June 7, 2020

Correspondence: Chang Mo Moon

Department of Internal Medicine, Tissue Injury Defence Research Center, Ewha Womans University College of Medicine, 1071 Anyangcheon-ro, Yangcheon-gu, Seoul 07985, Korea

Tel: +82-2-2650-2632, Fax: +82-2-2655-2076, E-mail: mooncm27@ewha.ac.kr ORCID: https://orcid.org/0000-0003-2550-913X

Ki-Nam Shim

Department of Internal Medicine, Ewha Womans University College of Medicine, 1071 Anyangcheon-ro, Yangcheon-gu, Seoul 07985, Korea

Tel: +82-2-2650-2632, Fax: +82-2-2655-2076, E-mail: shimkn@ewha.ac.kr

ORCID: https://orcid.org/0000-0003-4004-6292

cc This is an Open Access article distributed under the terms of the Creative Commons Attribution Non-Commercial License (http://creativecommons.org/ licenses/by-nc/3.0) which permits unrestricted non-commercial use, distribution, and reproduction in any medium, provided the original work is properly cited.

\section{INTRODUCTION}

The prevalence of iron deficiency anemia (IDA) is estimated to be $2 \%-5 \%$ in adult men and postmenopausal women. ${ }^{1}$ Because IDA is associated with high mortality and morbidity, as well as impaired quality of life, ${ }^{2}$ investigations to identify the underlying causes should be appropriately conducted. Although IDA has several etiologies, blood loss from the gastrointestinal (GI) tract is the most common cause in adult men and postmenopausal women. ${ }^{3}$ Therefore, esophagogastroduodenoscopy (EGD) and colonoscopy are performed after excluding an extra-GI bleeding focus through careful history 
taking, basic laboratory tests, and gynecological examination, particularly in premenopausal women. ${ }^{4}$ Subsequently, if EGD and colonoscopy are negative, capsule endoscopy (CE) is considered to evaluate the cause of obscure GI bleeding (OGIB), which is defined as persistent or recurrent bleeding without the identification of a definite bleeding focus in the initial evaluation. ${ }^{6}$

CE provides a noninvasive visualization of the entire small-bowel mucosa and is now recognized as the gold standard test for small-bowel investigation. ${ }^{7}$ On the basis of the Korean guideline for $\mathrm{CE}$ in $\mathrm{OGIB},{ }^{3} \mathrm{CE}$ could be an effective initial diagnostic tool for evaluating the cause of OGIB, with a higher diagnostic yield than small-bowel barium radiography, enteroclysis, computed tomography angiography, computed tomography enteroclysis, push enteroscopy, and double-balloon enteroscopy. ${ }^{3}$ The diagnostic yield of CE in OGIB has been reported to be $32 \%-83 \% .{ }^{8} \mathrm{CE}$ is also recommended as a primary diagnostic method for patients with IDA without an extra-GI bleeding focus. ${ }^{3}$

Although several studies have reported the clinical efficacy of CE in IDA and/or OGIB, data on the impact of CE in patients with unexplained IDA without overt GI bleeding are limited. Furthermore, most previous studies were designed as a single-center investigation with a relatively small number of patients. Therefore, this study aimed to evaluate the diagnostic yield of CE in patients with unexplained IDA who did not show overt bleeding, and to evaluate their long-term outcomes and related clinical factors, using a large CE registry in Korea.

\section{MATERIALS AND METHODS}

\section{Study subjects and data collection}

The Capsule Endoscopy Nationwide Database Registry (CAPENTRY), by the Korean Gut Image Study Group of the Korean Society of Gastrointestinal Endoscopy, includes cases performed in tertiary hospitals, which is estimated to cover a third of the entire cases. ${ }^{8}$ After CE examinations, the patients' data are registered into a web-based case report form by each corresponding investigator. By using the CE registry from January 2003 to February 2012, we selected prospectively collected data from patients aged $>18$ years who underwent $\mathrm{CE}$ for the evaluation of anemia after a complete evaluation with EGD and colonoscopy. Among these patients, those who satisfied the diagnostic criteria for IDA based on medical records were finally included. Subjects were excluded according to the following criteria: (1) overt GI bleeding, such as melena and hematochezia; (2) any positive result of active or/and recent bleeding stigmata in EGD or colonoscopy before CE; (3) history of inflammatory bowel disease; (4) extraintestinal conditions related to IDA, such as nasal bleeding, gynecological problem, or hematologic disorders; (5) poor-quality examination (unreliable examination in which significant fluid or debris disturbed the visualization by $>35 \%$ of the small-bowel transit time). ${ }^{9}$

Information, including age, sex, date of $\mathrm{CE}$, reason for $\mathrm{CE}$, $\mathrm{CE}$ diagnosis, quality of bowel preparation, $\mathrm{CE}$ findings, complete examination, and retention of $\mathrm{CE}$, was collected through an Internet website. In addition, the investigators involved in this study were instructed to collect the following demographic and clinical data from medical records: comorbidities, history of abdominal surgery, drug history, laboratory findings at the time of IDA diagnosis, lowest hemoglobin $(\mathrm{Hb})$ level in the past 3 months of $\mathrm{CE}$, fecal occult blood test (FOBT), findings of EGD and colonoscopy before $\mathrm{CE}$, treatment course after $\mathrm{CE}$, drug use after $\mathrm{CE}$, episode of GI bleeding after $\mathrm{CE}$, and follow-up duration to October 2015. The detailed information about the types of FOBT is shown in Supplementary Table 1.

Informed consent for the $\mathrm{CE}$ procedure and enrollment into the Korean Capsule Endoscopy Registry were obtained from all patients. All data were anonymized before the analysis. The study protocol was based on the Declaration of Helsinki and approved by the institutional review board of Ewah Womans University Hospital (approval number/date: 2014-10-16/December 27, 2014).

\section{Assessment and definitions}

$\mathrm{CE}$ findings were classified as vascular lesion, mucosal inflammatory lesion, and tumor lesion, ${ }^{10}$ and each lesion was evaluated with respect to whether it was clinically significant as a definite or probable origin of IDA. Angiodysplasia, active ulcer, active bleeding without an identified origin, and small-bowel tumor were defined as significant lesions, whereas venous ectasia, insignificant erosion or ulcer, erythematous mucosa, nonbleeding polyp or submucosal tumor, and hemangioma were defined as insignificant lesions. ${ }^{8}$ The diagnostic yield of $\mathrm{CE}$ was calculated as the number of identified significant lesions divided by the number of total CE examinations.

Treatment after CE was classified as interventional or noninterventional. Interventional treatment included endoscopic or surgical management, whereas noninterventional treatment included disease-specific medical treatment, medication adjustment, symptomatic control, and close observation. The primary endpoint was subsequent GI bleeding, defined as the presence of evidence of occult or overt GI bleeding after CE. We collected and assessed data on the clinical status of patients and their medication related to clinical outcomes (anti-coagulant or anti-platelet agent, nonsteroidal anti-inflammatory drugs [NSAIDs]) during the follow-up. 


\section{Statistical analysis}

All statistical analyses were performed using SPSS version 22 (SPSS Inc., Chicago, IL, USA). Statistical results are presented as number (\%) or mean \pm standard deviation. Student's $t$-test was used for continuous variables, and the chi-square test, Fisher's exact test, or linear-by-linear association was used for categorical variables. The Kaplan-Meier method and logrank test were performed to identify clinical variables at the initial CE associated with the outcome of interest: subsequent GI bleeding after CE. These variables included age $(\leq 60$ years vs. $>60$ years), sex, comorbidity, duration of IDA, $\mathrm{Hb}$ level, FOBT, anti-platelet medication before CE, CE findings (negative, insignificant, or significant) Subsequently, Cox regression analysis including the aforementioned variables was performed to determine the independent predictive factors for this outcome. $P$-values $<0.05$ were considered statistically significant.

\section{RESULTS}

\section{Baseline characteristics}

This study included 144 patients from 10 tertiary care academic medical institutions (Fig. 1). The baseline characteristics of the study subjects are shown in Table 1 . The mean age of the study population was $49.5 \pm 20.2$ years, and $50.7 \%$ were men. Approximately $19 \%$ of the patients had a history of hypertension, and $2.8 \%, 9 \%$, and $6.9 \%$ had taken anti-platelet agents, anti-coagulation agents, and NSAIDs, respectively. Approximately $33 \%$ of the patients had positive FOBT results. The mean duration of IDA was 9 months, and the patients were followed up for $17.8 \pm 28.9$ months.
Table 1. Baseline Characteristics of the Enrolled Patients

\begin{tabular}{lc}
\hline Patients ( $\boldsymbol{n = 1 4 4 )}$ & \\
\hline Age at diagnosis (yr) & $49.5 \pm 20.2$ \\
Male & $73(50.7)$ \\
Comorbidities & $27(18.8)$ \\
Hypertension & $14(9.7)$ \\
Diabetes mellitus & $3(2.1)$ \\
End stage renal disease & $3(2.1)$ \\
Cirrhosis & $4(2.8)$ \\
Heart failure & $0.2 \pm 0.7$ \\
Charlson comorbidity index & \\
Medication & $4(2.8)$ \\
Anti-coagulation agent & $13(9.0)$ \\
Anti-platelet agent & $10(6.9)$ \\
NSAIDs & \\
FOBT & \\
Positive & $35(32.7)$ \\
Negative & $72(67.3)$ \\
Lowest Hb during past 3 mo (gm/dL) & $7.2 \pm 2.1$ \\
Hb at CE (gm/dL) & $9.0 \pm 1.9$ \\
Follow-up duration (mo) & $17.8 \pm 28.9$ \\
\hline
\end{tabular}

Quantitative variables were expressed as mean \pm standard deviation. Categorical variables were expressed as number (\%).

CE, capsule endoscopy; FOBT, fecal occult blood test; Hb, hemoglobin; NSAIDs, nonsteroidal anti-inflammatory drugs.

${ }^{\text {a) }}$ FOBT was performed in 107 patients.

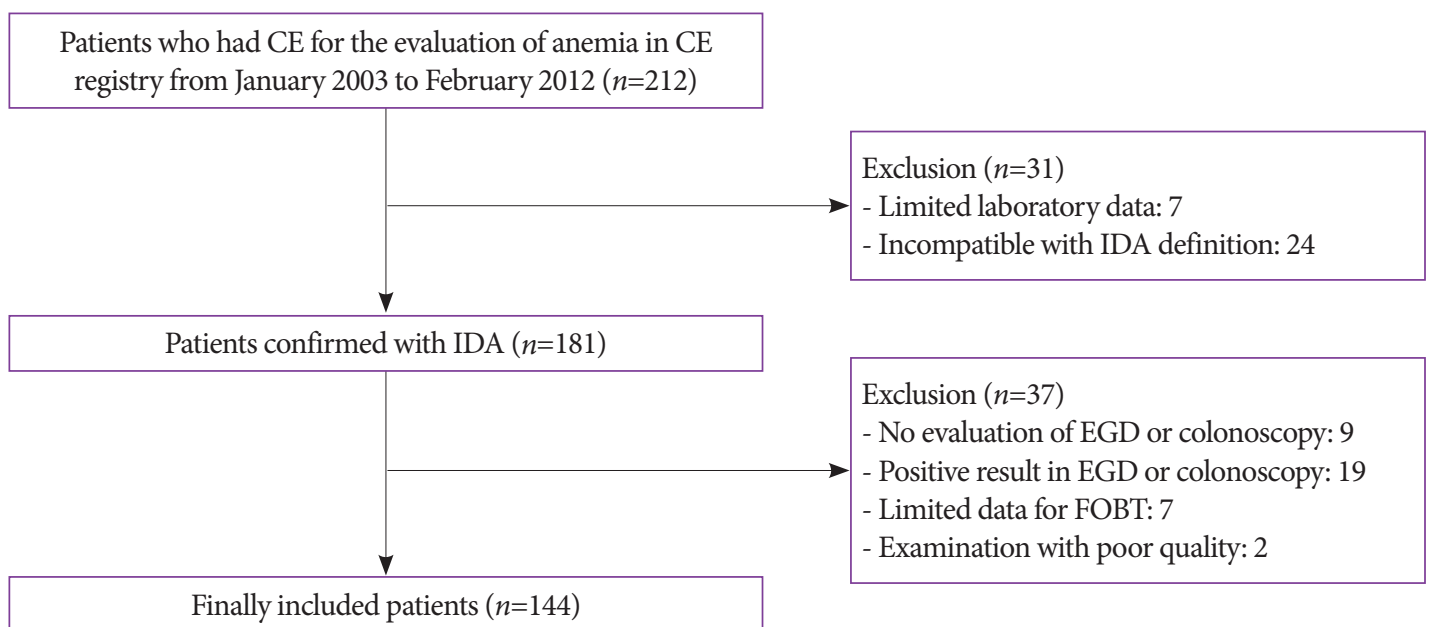

Fig. 1. Flowchart of the study subjects. CE, capsule endoscopy; EGD, esophagogastroduodenoscopy; FOBT, fecal occult blood test; IDA, iron deficiency anemia. 


\section{Findings of capsule endoscopy and clinical features}

The interval from the diagnosis of IDA to CE was $6.9 \pm 24.0$ months. The details of the CE findings are shown in Table 2. Lesions were detected in $56.2 \%$ of CE examinations, whereas $43.8 \%$ of the examinations had negative results. The most common finding was mucosal inflammatory lesion (23.6\%) followed by vascular lesion (20.8\%) and tumorous lesion (10.4\%), whereas angiodysplasia or venous ectasia was the most common disease entity (19.4\%), accounting for the majority of the vascular lesions. Among these 81 lesions found on $\mathrm{CE}$, clinically significant lesions were found in 49 patients, producing a diagnostic yield of $34 \%$. FOBT positivity had a significant association with distal small-bowel lesions. Among patients with positive FOBT results, $60 \%$ had distal small-bowel lesions $(p=0.017)$. The location of bleeding lesions in patients

Table 2. Findings of Capsule Endoscopy and Intervention

\begin{tabular}{lc}
\hline Finding of capsule endoscopy & $\boldsymbol{n}=\mathbf{1 4 4}$ \\
\hline Negative & $63(43.8)$ \\
Vascular lesions & $30(20.8)$ \\
Angiodysplasia & $26(18.0)$ \\
Bleeding of unidentified origin & $2(1.4)$ \\
Venous ectasia & $2(1.4)$ \\
\hline Mucosal inflammatory lesions & $34(23.6)$ \\
Non-specific erosion & $8(5.6)$ \\
Non-specific ulcer & $8(5.6)$ \\
Drug induced enteritis & $11(7.6)$ \\
Crohn's disease & $5(3.4)$ \\
Ulcerative colitis & $1(0.7)$ \\
Intestinal Behcet's disease & $1(0.7)$ \\
Tumorous lesions & $15(10.4)$ \\
Non-neoplastic polyp & $5(3.4)$ \\
Submucosal tumor & $6(4.2)$ \\
Neoplastic tumor & $3(2.1)$ \\
Hemangioma & $1(0.7)$ \\
Others & $2(1.4)$ \\
Capsule retention & $135(93.7)$ \\
No & $9(6.3)$ \\
Yes & \\
\hline Endervention & $8(5.6)$ \\
\hline Surgical & \\
\hline
\end{tabular}

Categorical variables were expressed as number (\%).

${ }^{a)}$ Others included one case of lymphangiectasia and congestive enteropathy, respectively. with positive FOBT results is shown in Supplementary Table 2. Nine patients $(6.3 \%)$ had capsule retention. The detailed information of patients with capsule retention is summarized in Supplementary Table 3.

On the basis of the CE findings, 1 patient $(0.7 \%)$ underwent endoscopic intervention and 8 patients $(5.6 \%)$ underwent surgical intervention, whereas 135 patients (93.7\%) received noninterventional treatment. The clinical outcomes of nine patients who underwent interventional treatment are summarized in Supplementary Table 4.

\section{Long-term outcome and prognostic factors}

During a mean follow-up of 17.8 months, GI bleeding was found in nine patients (6.3\%) after CE, with four (2.8\%) and five $(3.5 \%)$ patients presenting with occult bleeding and overt bleeding, respectively. The bleeding rate among those with significant lesions was $12.2 \%$ (6 of 49 patients; 3 patients with angiodysplasia, 1 patient with bleeding of unidentified origin, 2 patients diagnosed with inflammatory bowel disease). The bleeding rate in patients with insignificant lesions was 3.2\% (3 of 95), and all of these patients showed negative findings. Six patients who had significant lesions in CE were found to have GI bleeding that was attributed to small-bowel bleeding. Conversely, cases with insignificant findings in $\mathrm{CE}$ were found to have no definite small-bowel bleeding. GI bleeding was found in one patient $(20 \%)$ who took an anti-coagulant and in two patients (25\%) who took an anti-platelet after CE. No patient took NSAIDs at the time of GI bleeding. The mean interval from the time of CE to GI bleeding was 16.7 months (Table 3).

When the GI bleeding rate was analyzed using the KaplanMeier method, sex $(p=0.533)$, age $(p=0.486)$, comorbidity $(p=0.388)$, duration of anemia $(p=0.430)$, lowest Hb level $(p=0.789)$, use of an anti-platelet agent before CE $(p=0.104)$, significant findings on CE $(p=0.070)$, and intervention $(p=0.674)$ did not have a significant influence (Supplementary Fig. 1). However, patients with a positive FOBT result $(p=0.004)$ at the initial diagnosis had a higher rate of GI bleeding during the follow-up period (Fig. 2).

During the follow-up period, nine patients (three patients with occult bleeding and six patients with overt bleeding) showed GI bleeding after CE. In addition, a positive FOBT result was the only significant predictive factor for GI bleeding after CE in univariate Cox analysis (hazard ratio [HR], 5.58; 95\% confidence interval [CI], 1.49-20.89; $p=0.011$ ). Other findings, including sex, age, Charlson comorbidity index, duration of IDA, lowest $\mathrm{Hb}$ level, use of an anti-platelet agent before $\mathrm{CE}$, and intervention, were not associated with bleeding (Table 4). In multivariate analysis including the FOBT result and CE findings, a positive FOBT result (HR, 5.30; CI, 1.41$19.85 ; p=0.013$ ) was the only independent predictor. 
Table 3. Clinical Outcomes of Patients with Gastrointestinal Bleeding after Capsule Endoscopy

\begin{tabular}{|c|c|c|c|c|c|c|c|c|c|c|}
\hline No. & Sex & $\begin{array}{l}\text { Age } \\
(y r)\end{array}$ & $\begin{array}{l}\text { Initial CE find- } \\
\text { ings }\end{array}$ & $\begin{array}{l}\text { Initial } \\
\text { FOBT } \\
\text { result }\end{array}$ & Medication & $\begin{array}{c}\text { Interval } \\
\text { from CE to } \\
\text { GI bleeding } \\
\text { (mo) }\end{array}$ & $\begin{array}{c}\text { Type } \\
\text { of GI } \\
\text { bleeding }\end{array}$ & $\begin{array}{l}\text { Hb at GI } \\
\text { bleeding } \\
(\mathrm{gm} / \mathrm{dL})\end{array}$ & $\begin{array}{l}\text { Hospital- } \\
\text { ization } \\
\text { due to GI } \\
\text { bleeding }\end{array}$ & Diagnosis at GI bleeding \\
\hline 1 & $\mathrm{~F}$ & 71 & Negative & + & None & 3 & Occult & 13.0 & No & $\begin{array}{l}\text { R/O descending colon } \\
\text { diverticular bleeding }\end{array}$ \\
\hline 2 & $\mathrm{~F}$ & 75 & Negative & - & None & 24 & Overt & 6.0 & Yes & Active gastric ulcer \\
\hline 3 & $\mathrm{~F}$ & 59 & Negative & + & $\begin{array}{l}\text { Anticoagula- } \\
\text { tion }\end{array}$ & 49 & Overt & 3.0 & - & Unknown $^{\text {a) }}$ \\
\hline 4 & $\mathrm{M}$ & 73 & Angiodysplasia & + & Aspirin & 2 & Occult & 10.3 & No & $\begin{array}{c}\text { Recurrent angiodysplasia } \\
\text { bleeding }\end{array}$ \\
\hline 5 & $\mathrm{~F}$ & 67 & Angiodysplasia & + & None & 3 & Occult & 7.1 & Yes & $\begin{array}{l}\text { Portal hypertensive en- } \\
\text { teropathy }\end{array}$ \\
\hline 6 & M & 61 & Angiodysplasia & + & Aspirin & 36 & Overt & 13 & Yes & $\begin{array}{c}\text { Anastomosis site bleeding } \\
\text { of ileocolostomy }\end{array}$ \\
\hline 7 & $\mathrm{M}$ & 18 & $\begin{array}{c}\text { Bleeding of } \\
\text { unidentified } \\
\text { origin }\end{array}$ & + & None & 28 & Overt & 14.3 & Yes & $\begin{array}{c}\text { Newly diagnosed CD (jeju- } \\
\text { nal bleeding) }\end{array}$ \\
\hline 8 & $\mathrm{~F}$ & 55 & $\mathrm{CD}$ & Unknown & None & 1 & Overt & 10.6 & Yes & $\begin{array}{l}\text { Aggravation of CD } \\
\text { (colon bleeding) }\end{array}$ \\
\hline 9 & $\mathrm{M}$ & 22 & $\mathrm{CD}$ & + & None & 4 & Occult & 7.4 & No & $\begin{array}{c}\text { Aggravation of CD } \\
\text { (jejunal and ileal bleeding) }\end{array}$ \\
\hline
\end{tabular}

CD, Crohn's disease; CE, capsule endoscopy; FOBT, fecal occult blood test; GI, gastrointestinal; Hb, hemoglobin; R/O, rule out.

a) The data of this patient could not be acquired because this patient transferred to other hospital that was not related to this study for evaluation of GI bleeding.

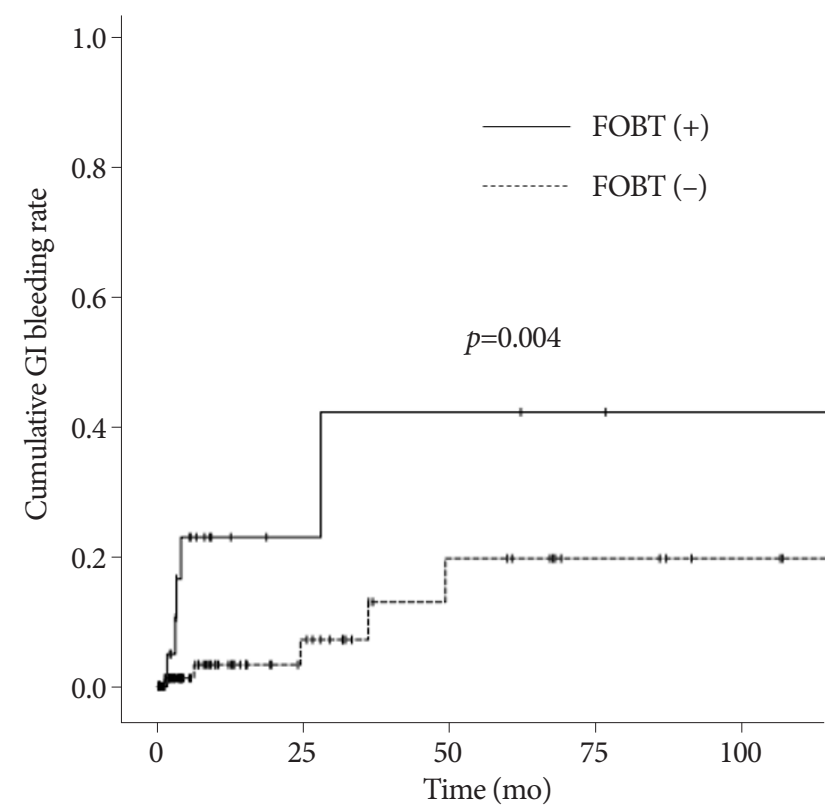

Fig. 2. Kaplan-Meier curve showing the cumulative gastrointestinal (GI) bleeding rate according to fecal occult blood test (FOBT) result. 
Table 4. Pridictive Factors Associated with Gastrointestinal Bleeding after Capsule Endoscopy

\begin{tabular}{|c|c|c|c|}
\hline & Harzard ratio & $95 \% \mathrm{CI}$ & $p$-value \\
\hline Female & 1.53 & $0.40-5.84$ & 0.536 \\
\hline Age $>60$ & 1.59 & $0.43-5.97$ & 0.490 \\
\hline Charlson comorbidity index & 2.30 & $0.91-5.80$ & 0.077 \\
\hline Duration of IDA $>6 \mathrm{mo}$ & 0.44 & $0.06-3.54$ & 0.442 \\
\hline Lowest $\mathrm{Hb}$ level < $8 \mathrm{~g} / \mathrm{dL}$ & 1.24 & $0.26-5.98$ & 0.789 \\
\hline Positve FOBT & 5.58 & $1.49-20.89$ & 0.011 \\
\hline Anti-platelets before CE & 3.04 & $0.74-12.39$ & 0.122 \\
\hline \multicolumn{4}{|l|}{ CE findings } \\
\hline Negative or insignificant ${ }^{\mathrm{a})}$ & 1 & & \\
\hline Significant ${ }^{\mathrm{b})}$ & 3.34 & $0.84-13.37$ & 0.088 \\
\hline Intervention $^{c}$ & 0.04 & $0.0-101.45$ & 0.416 \\
\hline
\end{tabular}

CE, capsule endoscopy; CI, confidence interval; FOBT, fecal occult blood test; Hb, hemoglobin; IDA, iron deficiency anemia.

${ }^{a)}$ Insignificant lesions included venous ectasia, non-specific erosion or ulcer, erythematous mucosa, non-bleeding polyp, submucosal tumor, and hemangioma.

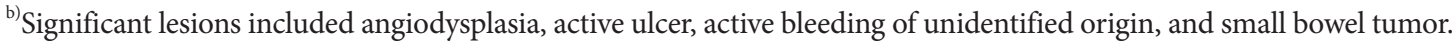

${ }^{c}$ Interventional treatment included endoscopic or surgical management.

\section{DISCUSSION}

This was a nationwide registry-based study of CE data from 10 tertiary hospitals in Korea that focused on the diagnostic yield of CE in patients with unexplained IDA without overt GI bleeding. The present study demonstrated that the diagnostic yield of $\mathrm{CE}$ was $34 \%$, which was lower than that reported in previous studies that focused on overt OGIB $(88 \%-92.3 \%))^{10,11}$ In addition, a positive FOBT result was an independent predictive factor for subsequent GI bleeding after $\mathrm{CE}$, whereas the presence of clinically significant lesions and the application of interventional treatment did not influence this outcome.

IDA is a frequently encountered condition in clinical practice and accounts for $13 \%$ of outpatient cases referred to gastroenterologists. ${ }^{12}$ The most frequent cause of IDA is gynecological disorder in premenopausal women, and GI disorder in postmenopausal women and men. ${ }^{13,14}$ Some authors have suggested that all otherwise unexplained IDA should be evaluated as OGIB. ${ }^{15}$ OGIB is defined as recurrent or persistent bleeding of an unknown etiology after obtaining negative EGD and colonoscopy results. ${ }^{16}$ OGIB accounts for $5 \%$ of all GI bleeding and is further subdivided into the overt and occult types based on the passage of visible blood. ${ }^{17}$ Because small-bowel pathology accounts for $75 \%$ of OGIB, small-bowel evaluation is necessary in OGIB. ${ }^{16,18}$

CE has contributed to advancements in the diagnosis of small-bowel lesions by providing a noninvasive and reliable small-intestinal imaging modality, thus gaining a reputation as an initial diagnostic method for OGIB evaluation. Although many studies have reported the clinical impact of CE in OGIB, most of those studies focused on overt OGIB, whereas only a few studies evaluating occult OGIB reported a lower diagnostic yield than that for overt OGIB. ${ }^{19-21}$ The overall diagnostic yield of CE in OGIB has been reported to broadly range from $32 \%$ to $83 \%$ based on the bleeding type. ${ }^{19,21-23}$ The diagnostic yield was higher in those with overt bleeding $(60 \%-67 \%)^{19,24}$ than in those with occult bleeding $(44 \%-48 \%){ }^{10,11,24}$ With respect to the diagnostic yield of CE in IDA, a meta-analysis reported an overall diagnostic yield of $47 \%$; however, subgroup analyses showed different positive yields when solely patients with IDA were evaluated (66\%) and when patients with IDA and those with other conditions were investigated (44\%). ${ }^{25} \mathrm{~A}$ Canadian study also reported the diagnostic yield as $45.5 \%$ in patients with IDA without evidence of overt or occult GI bleeding. ${ }^{26}$ Compared with the previous study, our diagnostic yield, namely $34 \%$, seems slightly lower. However, this discrepancy could be attributed to the study design or study subjects. We included patients with unexplained IDA without overt bleeding and excluded those with preexisting clinical conditions that could be a potential source of OGIB in our study, whereas the Canadian study adjusted only for dietary factor and menorrhagia. In addition, the method of interpretation of positive lesions could be another reason because no standard definition exists for defining the diagnostic yield of 
CE in OGIB. Although we defined only highly relevant (P2) lesions as significant lesions, previous studies included lesions that could result in changes in further management or all detected erosion and ulcers. Furthermore, the heterogeneity in age, ${ }^{11}$ comorbidity, and concurrent medication, ${ }^{27}$ which have been suggested as factors associated with positive CE findings, could affect the diagnostic yield. Thus, a careful interpretation based on the study design and characteristics of study subjects is required.

With respect to the predictive factor for GI bleeding after $\mathrm{CE}$, positive FOBT was a significant predictor for this outcome in our study. To our knowledge, no study has evaluated the risk factor for GI bleeding after CE in patients with IDA. Most studies on CE in patients with IDA have focused only on the diagnostic yield of $\mathrm{CE}$, and only a few studies evaluating OGIB reported the long-term outcome after CE. Depending on the type of bleeding investigated, several different factors have been proposed as a predictive factor for rebleeding. A recent Korean multicenter study based on the same CE registry as that used in our study, excluding cases without a definite evidence of GI bleeding, reported that the presence of angiodysplasia and an OGIB duration of $>3$ months were significant factors for rebleeding after CE. ${ }^{8}$ Although this study is a well-designed, multicenter study, only $11.5 \%$ of patients had occult OGIB, which could be a limiting factor for a direct comparison with our study. A prospective study performed in Greece, demonstrating similar proportions of overt and occult OGIB, reported that patients with positive findings on CE had a higher rate of complete resolution of bleeding than patients with negative finding. ${ }^{28}$ However, angiodysplasia accounted for a major portion (70\%) of the CE-positive group and the mean follow-up duration was relatively short at 5.2 months. Although there are very limited studies evaluating the clinical impact of CE on occult OGIB, a recent Japanese study reported that the prevalence of small-bowel lesions was higher in the positive FOBT group than in the negative FOBT group among patients with occult OGIB, whereas the prevalence was not different based on FOBT results in patients with overt OGIB. This study also showed the importance of CE timing on the diagnostic yield of $\mathrm{CE}$, namely within a day after FOBT. ${ }^{21}$ Although this study did not evaluate the long-term outcomes after CE, it suggested the importance of FOBT in diagnosing and managing occult OGIB. Taking into account the results of previous studies and our results, the differences in the inclusion criteria for the study subjects, in the proportion of significant lesions, and in the intervention after CE may have led to different results.

This study had several limitations. First, although this study evaluated prospectively collected data from a CE registry, a bias could exist or the information may be limited because these data were analyzed in a retrospective manner. For example, the information on whether the CE readers were blinded to the indication of $\mathrm{CE}$, which might influence the diagnosis, could not be accessed. Moreover, some bleeding cases may have been missed in asymptomatic patients because not all patients were evaluated for their symptoms or with FOBT in the specific period. However, considering that the mean follow-up duration was 17.8 months and most patients were followed up in the asymptomatic state, we believe that the chance of missing clinically significant bleeding cases was low. Second, because the $\mathrm{CE}$ registry includes only cases from tertiary hospitals (accounting for a third of all cases), the patients included in this study could not represent the characteristics of the general population, thus resulting in a selection bias. However, considering that most patients with unexplained IDA tend to be referred to tertiary hospitals for further evaluation, the selection bias would not cause a significant difference. Lastly, although we attempted to collect all available data from the 10 hospitals to recruit a sufficient number of patients for the analysis, the relatively small number of the analyzed patients could be a limitation of this study. Despite these limitations, our study is valuable in terms of being the first study to focus on patients with unexplained IDA without overt bleeding in Korea.

In conclusion, the present study demonstrates a $34 \%$ overall diagnostic yield for $\mathrm{CE}$ in patients with unexplained IDA without overt GI bleeding. Because positive FOBT at the initial diagnosis is an independent predictive factor for GI bleeding during the follow-up after $\mathrm{CE}$, patients with positive FOBT need to be more closely followed up.

Conflicts of Interest

The authors have no financial conflicts of interest.

Author Contributions

Conceptualization: Chang Mo Moon, Ki-Nam Shim

Data curation: Ji Young Chang, Dae Young Cheung, Hyun Seok Lee, Yun Jeong Lim, Seong Ran Jeon, Soo Jung Park, Kyeong Ok Kim, Hyun Joo Song, Hyun Joo Jang, Ji Hyun Kim

Formal analysis: JYC

Investigation: JYC, DYC, HSL, YJL, SRJ, SJP, KOK, HJS, HJJ, JHK

Methodology: CMM, KNS

Supervision: CMM, KNS

Validation: CMM, KNS

Writing-original draft: JYC

Writing-review\&editing: JYC

ORCID

Ji Young Chang: https://orcid.org/0000-0002-7951-456X

Dae Young Cheung: https://orcid.org/0000-0003-4150-3555

Hyun Seok Lee: https://orcid.org/0000-0001-9288-0321

Yun Jeong Lim: https://orcid.org/0000-0002-3279-332X

Seong Ran Jeon: https://orcid.org/0000-0001-6970-9737

Soo Jung Park: https://orcid.org/0000-0003-0699-6809 
Kyeong Ok Kim: https://orcid.org/0000-0001-5799-7436

Hyun Joo Song: https://orcid.org/0000-0002-2561-555X

Hyun Joo Jang: https://orcid.org/0000-0003-4424-1968

Ji Hyun Kim: https://orcid.org/0000-0003-0861-2792

\section{REFERENCES}

1. Guralnik JM, Eisenstaedt RS, Ferrucci L, Klein HG, Woodman RC. Prevalence of anemia in persons 65 years and older in the United States: evidence for a high rate of unexplained anemia. Blood 2004;104:22632268.

2. Nissenson AR, Goodnough LT, Dubois RW. Anemia: not just an innocent bystander? Arch Intern Med 2003;163:1400-1404.

3. Shim KN, Moon JS, Chang DK, et al. Guideline for capsule endoscopy: obscure gastrointestinal bleeding. Clin Endosc 2013;46:45-53.

4. De Franceschi L, Iolascon A, Taher A, Cappellini MD. Clinical management of iron deficiency anemia in adults: systemic review on advances in diagnosis and treatment. Eur J Intern Med 2017;42:16-23.

5. Fireman Z, Kopelman Y. The role of video capsule endoscopy in the evaluation of iron deficiency anaemia. Dig Liver Dis 2004;36:97-102.

6. Zuckerman GR, Prakash C, Askin MP, Lewis BS. AGA technical review on the evaluation and management of occult and obscure gastrointestinal bleeding. Gastroenterology 2000;118:201-221.

7. Holleran GE, Barry SA, Thornton OJ, Dobson MJ, McNamara DA. The use of small bowel capsule endoscopy in iron deficiency anaemia: low impact on outcome in the medium term despite high diagnostic yield. Eur J Gastroenterol Hepatol 2013;25:327-332.

8. Min YW, Kim JS, Jeon SW, et al. Long-term outcome of capsule endoscopy in obscure gastrointestinal bleeding: a nationwide analysis. Endoscopy 2014;46:59-65.

9. Niv Y, Niv G, Wiser K, Demarco DC. Capsule endoscopy - comparison of two strategies of bowel preparation. Aliment Pharmacol Ther 2005;22:957-962.

10. Arakawa D, Ohmiya N, Nakamura M, et al. Outcome after enteroscopy for patients with obscure GI bleeding: diagnostic comparison between double-balloon endoscopy and videocapsule endoscopy. Gastrointest Endosc 2009;69:866-874.

11. Pennazio M, Santucci R, Rondonotti E, et al. Outcome of patients with obscure gastrointestinal bleeding after capsule endoscopy: report of 100 consecutive cases. Gastroenterology 2004;126:643-653.

12. McIntyre AS, Long RG. Prospective survey of investigations in outpatients referred with iron deficiency anaemia. Gut 1993;34:1102-1107.

13. Milano A, Balatsinou C, Filippone A, et al. A prospective evaluation of iron deficiency anemia in the GI endoscopy setting: role of standard endoscopy, videocapsule endoscopy, and CT-enteroclysis. Gastrointest
Endosc 2011;73:1002-1008.

14. Bermejo F, García-López S. A guide to diagnosis of iron deficiency and iron deficiency anemia in digestive diseases. World J Gastroenterol 2009;15:4638-4643.

15. Rockey DC. Occult and obscure gastrointestinal bleeding: causes and clinical management. Nat Rev Gastroenterol Hepatol 2010;7:265-279.

16. Raju GS, Gerson L, Das A, Lewis B. American Gastroenterological Association (AGA) institute medical position statement on obscure gastrointestinal bleeding. Gastroenterology 2007;133:1694-1696.

17. Law R, Varayil JE, WongKeeSong LM, et al. Assessment of multi-modality evaluations of obscure gastrointestinal bleeding. World J Gastroenterol 2017;23:614-621.

18. ASGE Standards of Practice Committee, Fisher L, Lee Krinsky M, et al. The role of endoscopy in the management of obscure GI bleeding. Gastrointest Endosc 2010;72:471-479.

19. Carey EJ, Leighton JA, Heigh RI, et al. A single-center experience of 260 consecutive patients undergoing capsule endoscopy for obscure gastrointestinal bleeding. Am J Gastroenterol 2007;102:89-95.

20. Ell C, Remke S, May A, Helou L, Henrich R, Mayer G. The first prospective controlled trial comparing wireless capsule endoscopy with push enteroscopy in chronic gastrointestinal bleeding. Endoscopy 2002;34:685689.

21. Kobayashi Y, Watabe H, Yamada A, et al. Impact of fecal occult blood on obscure gastrointestinal bleeding: observational study. World J Gastroenterol 2015;21:326-332.

22. Concha R, Amaro R, Barkin JS. Obscure gastrointestinal bleeding: diagnostic and therapeutic approach. J Clin Gastroenterol 2007;41:242-251.

23. Calabrese C, Liguori G, Gionchetti P, et al. Obscure gastrointestinal bleeding: single centre experience of capsule endoscopy. Intern Emerg Med 2013;8:681-687.

24. Katsinelos P, Lazaraki G, Gkagkalis A, et al. The role of capsule endoscopy in the evaluation and treatment of obscure-overt gastrointestinal bleeding during daily clinical practice: a prospective multicenter study. Scand J Gastroenterol 2014;49:862-870.

25. Koulaouzidis A, Rondonotti E, Giannakou A, Plevris JN. Diagnostic yield of small-bowel capsule endoscopy in patients with iron-deficiency anemia: a systematic review. Gastrointest Endosc 2012;76:983-992.

26. Tong J, Svarta S, Ou G, Kwok R, Law J, Enns R. Diagnostic yield of capsule endoscopy in the setting of iron deficiency anemia without evidence of gastrointestinal bleeding. Can J Gastroenterol 2012;26:687-690.

27. Sidhu R, Sanders DS, Kapur K, Leeds JS, McAlindon ME. Factors predicting the diagnostic yield and intervention in obscure gastrointestinal bleeding investigated using capsule endoscopy. J Gastrointestin Liver Dis 2009;18:273-278.

28. Viazis N, Papaxoinis K, Vlachogiannakos J, Efthymiou A, Theodoropoulos I, Karamanolis DG. Is there a role for second-look capsule endoscopy in patients with obscure GI bleeding after a nondiagnostic first test? Gastrointest Endosc 2009;69:850-856. 